\title{
Dendritic Calcium Activity Precedes Inspiratory Bursts in preBötzinger Complex Neurons
}

\author{
Christopher A. Del Negro, ${ }^{1}$ John A. Hayes, ${ }^{1}$ and Jens C. Rekling ${ }^{2}$ \\ ${ }^{1}$ Department of Applied Science, McGlothlin-Street Hall, The College of William and Mary, Williamsburg, Virginia 23187, and 2 Department of Neuroscience \\ and Pharmacology, Panum Institute, Copenhagen DK-2200, Denmark
}

Medullary interneurons of the preBötzinger complex assemble excitatory networks that produce inspiratory-related neural rhythms, but the importance of somatodendritic conductances in rhythm generation is still incompletely understood. Synaptic input may cause $\mathrm{Ca}^{2+}$ accumulation postsynaptically to evoke a $\mathrm{Ca}^{2+}$-activated inward current that contributes to inspiratory burst generation. We measured $\mathrm{Ca}^{2+}$ transients by two-photon imaging dendrites while recording neuronal somata electrophysiologically. Dendritic $\mathrm{Ca}^{2+}$ accumulation frequently precedes inspiratory bursts, particularly at recording sites 50-300 $\mu \mathrm{m}$ distal from the soma. Preinspiratory $\mathrm{Ca}^{2+}$ transients occur in hotspots, not ubiquitously, in dendrites. $\mathrm{Ca}^{2+}$ activity propagates orthodromically toward the soma (and antidromically to more distal regions of the dendrite) at rapid rates $(300-700 \mu \mathrm{m} / \mathrm{s})$. These high propagation rates suggest that dendritic $\mathrm{Ca}^{2+}$ activates an inward current to electrotonically depolarize the soma, rather than propagate as a regenerative $\mathrm{Ca}^{2+}$ wave. These data provide new evidence that respiratory rhythmogenesis may depend on dendritic burst-generating conductances activated in the context of network activity.

\section{Introduction}

Breathing behavior in mammals depends on inspiratory neural rhythms that originate in the preBötzinger complex (preBötC) of the ventral medulla. Glutamatergic interneurons comprise its rhythmogenic core and a key unsolved question pertains to the spatiotemporal distribution of active conductances in the somatodendritic membrane and their role in rhythmogenesis (Ramirez et al., 2004; Feldman and Del Negro, 2006). One possible mechanism posits that recurrent synaptic interactions evoke postsynaptic conductances to generate inspiratory bursts (Rekling et al., 1996; Rekling and Feldman, 1998). Specifically, recurrent excitation may elevate postsynaptic $\mathrm{Ca}^{2+}$ and thus activate nonspecific cationic current $\left(I_{\mathrm{CAN}}\right)$ (Mironov, 2008; Rubin et al., 2009). Studies in a slice model of respiratory rhythm show that $I_{\text {CAN }}$ is one important charge carrier underlying inspiratory bursts (Thoby-Brisson and Ramirez, 2001; Peña et al., 2004) and that the synaptic recruitment of $I_{\mathrm{CAN}}$ takes place in the dendrites (Pace et al., 2007b; Mironov, 2008).

Glutamatergic preBötC neurons have the potential to excite their postsynaptic targets and thus cause $\mathrm{Ca}^{2+}$ to accumulate in the dendrites before inspiratory bursts. This has been

Received Sept. 9, 2010; revised Oct. 18, 2010; accepted Nov. 7, 2010.

This work was supported by Grants R01-HL104127-01 (to C.D.N.), The Danish Medical Council, the NovoNordisk Foundation, Den Owensenske Fond, Lægeforeningens forskningsfond, and Agnes og Pouls Friis Fond (to J.C.R.).

Correspondence should be addressed to Dr. Christopher A. Del Negro. Present address (until May 2011): Institut de Neurobiologie Alfred Fessard, Neurobiologie et Développement, Centre National de la Recherche Scientifique, Avenue de la terrasse, 91198 Gif-sur-Yvette, France. Permanent address: McGlothlin-Street Hall, Room 318, The College of William and Mary, Williamsburg, Virginia 23187-8795. E-mail: cadeln@wm.edu.

J. A. Hayes' present address: Institut de Neurobiologie Alfred Fessard, Neurobiologie et Développement Centre National de la Recherche Scientifique, 91198 Gif-sur-Yvette, France. E-mail: john.hayes@inaf.cnrs-gif.fr.

DOI:10.1523/JNEUROSCI.4731-10.2011

Copyright $\odot 2011$ the authors $\quad 0270-6474 / 11 / 311017-06 \$ 15.00 / 0$ examined in slices and organotypic cultures but recording sites were limited to proximal dendrites $(\sim 50 \mu \mathrm{m}$ from the soma) and many data were acquired from nonrhythmic cultures (Mironov, 2008, 2009). Therefore, the extent to which these data apply to preBötC neuron dendrites in the context of rhythmogenesis remains uncertain. We measured postsynaptic $\mathrm{Ca}^{2+}$ dynamics in soma-close and soma-distal dendritic locations, using acute slices that retain respiratory function in vitro. We show that endogenous synaptic drive evokes dendritic $\mathrm{Ca}^{2+}$ transients before somatic inspiratory bursts, which suggests that synaptically activated dendritic conductances may influence respiratory rhythm generation.

\section{Materials and Methods}

The Institutional Animal Care and Use Committee at the College of William and Mary and the Danish National Committee for Ethics in Animal Research approved all procedures. Neonatal mice [postnatal days (P) 0 -6] were dissected in artificial CSF (ACSF) containing the following (in mM): $124 \mathrm{NaCl}, 3 \mathrm{KCl}, 1.5 \mathrm{CaCl}_{2}, 1 \mathrm{MgSO}_{4}, 25 \mathrm{NaHCO}_{3}, 0.5$ $\mathrm{NaH}_{2} \mathrm{PO}_{4}$, and 30 dextrose, equilibrated with $95 \% \mathrm{O}_{2} / 5 \% \mathrm{CO}_{2}, \mathrm{pH} 7.4$. $\mathrm{CaCl}_{2}$ was adjusted to $1.1 \mathrm{~mm}$ in some experiments (see below). We cut $550-\mu \mathrm{m}$-thick transverse slices that exposed the preBötC at the rostral face (Ruangkittisakul et al., 2010) Slices were perfused with $27^{\circ} \mathrm{C}$ oxygenated ACSF at $4 \mathrm{ml} / \mathrm{min}$. The $\mathrm{K}^{+}$concentration $\left(\left[\mathrm{K}^{+}\right]_{\mathrm{o}}\right)$ was raised to 9 $\mathrm{mm}$ in ACSF containing $1.5 \mathrm{~mm} \mathrm{CaCl}_{2}$ and to $5 \mathrm{~mm}$ in ACSF containing $1.1 \mathrm{mM} \mathrm{CaCl}_{2}$. In both cases, the tradeoff of raising $\left[\mathrm{K}^{+}\right]_{\mathrm{o}}$ toward a maximum of $9 \mathrm{~mm}$ and lowering $\left[\mathrm{Ca}^{2+}\right]_{\mathrm{o}}$ toward a minimum of $1 \mathrm{~mm}$ maintained excitability and sustained rhythmic function (Ruangkittisakul et al., 2007). Motor output was recorded from hypoglossal (XII) nerve roots.

Infrared-enhanced differential interference contrast videomicroscopy was performed using a Zeiss fixed-stage microscope with Köhler illumination and a $20 \times / 1.0$ numerical aperture (NA) objective (Carl Zeiss). 
We performed whole-cell recordings using an EPC-10 amplifier (HEKA). Data were filtered at $1 \mathrm{kHz}$ and acquired at $10 \mathrm{kHz}$. Standard patch solution contained the following (in mM): $140 \mathrm{~K}$-Gluconate, $10 \mathrm{NaCl}, 0.5 \mathrm{CaCl}_{2}$, 10 HEPES, 1 EGTA, $2 \mathrm{Mg}$-ATP, and $0.3 \mathrm{Na} 3-$ GTP, pH 7.3. We added $200 \mu \mathrm{M}$ Oregon Green 488 BAPTA-2 (OGB-2; $\left.\mathrm{K}_{\mathrm{d}}=580 \mathrm{~nm}\right)$ and 50 $\mu \mathrm{M}$ Alexa 568 hydrazide (Invitrogen) before use.

We performed two-photon imaging using a Ti:Sapphire laser (Spectra Physics) in conjunction with a laser-scanning microscope (Zeiss). Images were acquired by non-descanned detectors at $20 \mathrm{~Hz}$.

We gated image-acquisition protocols via logic pulses from the EPC-10. Gating pulses were recorded at $10 \mathrm{kHz}$ in a separate channel of the chart recorder along with logic pulses returned by the LSM 510 upon the initiation of the first (and last) scans of the time series. Return pulses were used to sync the electrophysiology data with the image stack.

We report 54 neurons recorded with $200 \mu \mathrm{M}$ OGB-2. We varied the concentration and the affinity of the $\mathrm{Ca}^{2+}$ dye to discern optimal recording conditions, using $400 \mu \mathrm{M}$ Oregon Green 488 BAPTA-1 (OGB-1; $\mathrm{K}_{\mathrm{d}}=190 \mathrm{nM}$ ) and 100-500 $\mu \mathrm{M}$ Oregon Green 488 BAPTA-6F (OGB-6F; $\left.\mathrm{K}_{\mathrm{d}}=3 \mu \mathrm{M}\right)$ in preliminary experiments. OGB-6F yielded fast-decaying somatic signals, but the low affinity of the dye diminished dendritic fluorescence. OGB-1 was readily detectable in dendrites, but its long-lasting decay caused spurious temporal summation of fluorescence changes $(\Delta F)$. We report OGB-1 experiments wherein $\Delta F$ decayed to baseline during each respiratory cycle. The rise time was equally rapid for OGB-1, OGB-2, and OGB-6F.

Alexa 568 hydrazide dye in the patch solution enabled us to visualize the dendritic arbor within $5 \mathrm{~min}$ of establishing the whole-cell configuration. Three to six principle dendrites were always visible in preBötC neurons. OGB diffuses more slowly than Alexa 568 hydrazide, so we waited at least 20 min before measuring dendritic $\mathrm{Ca}^{2+}$ activity. After $20 \mathrm{~min}$, we recorded one principle dendrite per $10 \mathrm{~min}$ until whole-cell recording conditions deteriorated.

In eight experiments, we imaged the soma and proximal dendrites of preBötC neurons loaded with membrane-permeable $\mathrm{Ca}^{2+}$ dye in vitro. Slices were loaded with Fluo-8 AM $\left(\mathrm{K}_{\mathrm{d}}=389 \mathrm{~nm}\right.$; AAT Bioquest $)$ via submersion for 60-90 min while bubbling with $95 \% \mathrm{O}_{2} / 5 \% \mathrm{CO}_{2}$. We dissolved $50 \mu \mathrm{g}$ of Fluo-8 AM in $50 \mu \mathrm{l}$ of DMSO and then combined 20 $\mu \mathrm{l}$ of the Fluo- 8 AM solution with $5 \mu \mathrm{l}$ of cremophore EL (Fluka) and 5 $\mu \mathrm{l}$ of $20 \%$ Pluronic F-127 in DMSO. We added the $30 \mu \mathrm{l}$ of Fluo- 8 AM solution to $1 \mathrm{ml}$ of ACSF with $100 \mathrm{~mm}$ mannitol (Sigma-Aldrich) and 100 $\mu \mathrm{M}$ MK-571 (Enzo Life Sciences). The final Fluo-8 AM concentration was $20 \mu \mathrm{M}$.

We imaged Fluo-8 AM-labeled neurons using an Olympus BX51 microscope with a $63 \times / 0.95 \mathrm{NA}$ objective. Image stacks were acquired at 70 $\mathrm{Hz}$ using a CCD camera (Andor Technology).

Images were analyzed with Image $(\mathrm{NIH})$. For each bout, we created a composite image showing the SD of fluorescence through the image stack. This composite shows dendritic regions of interest (ROIs) with large $\Delta F$, which often indicates periodicity. For each ROI, we plotted a histogram of fluorescence measurements and fitted a Gaussian function to determine the mean and SD. The mean reflects baseline fluorescence $\left(F_{0}\right)$ and was used to normalize the $\Delta F$ signal, which was subsequently displayed as $\Delta F / F_{0}$. The SD was used to determine the onset of fluores- cence bursts. The first point in the $\Delta F / F_{0}$ signal to exceed baseline by $\geq 2$ SD was considered statistically significant at $p<0.05$ and was defined as the dendritic fluorescence onset time $\left(T_{\mathrm{DEN}}\right)$. The onset time of the somatic burst $\left(T_{\text {SOMA }}\right)$ was defined as the absolute time of maximum rate of rise $\left(d V_{M} / d t\right)$ of the voltage, or the maximum rate of rise of somatic $\mathrm{Ca}^{2+}$ fluorescence. The inherent disparity in sampling rate and signalto-noise ratio was the basis for defining $T_{\mathrm{DEN}}$ and $T_{\mathrm{SOMA}}$. Cycletriggered averages of inspiratory bursts were computed from $\geq 7$ consecutive cycles based on a threshold-crossing algorithm applied to the XII output. Dendritic $\mathrm{Ca}^{2+}$ latency $(\Delta T)$ was defined as the difference between $T_{\text {DEN }}$ and $T_{\text {SOMA }},\left(\Delta T=T_{\text {DEN }}-T_{\text {SOMA }}\right)$.

\section{Results}

We recorded preBötC interneurons showing evidence of a build-up of presynaptic excitatory input and strong inspiratory bursts, including summating EPSPs, ramp-like depolarization, and spike discharge at a low rate $(2-10 \mathrm{~Hz})$ during the expiratory phase, particularly 300-900 ms before XII output. Robust inspiratory bursts experience depolarization block (i.e., voltage-dependent spike inactivation), which attenuates intraburst spiking. We probed for these characteristics, which may indicate rhythmogenic function, during on-cell recording (Figs. $1 A, 2 A$ ) as selection criteria for subsequent wholecell recording. 

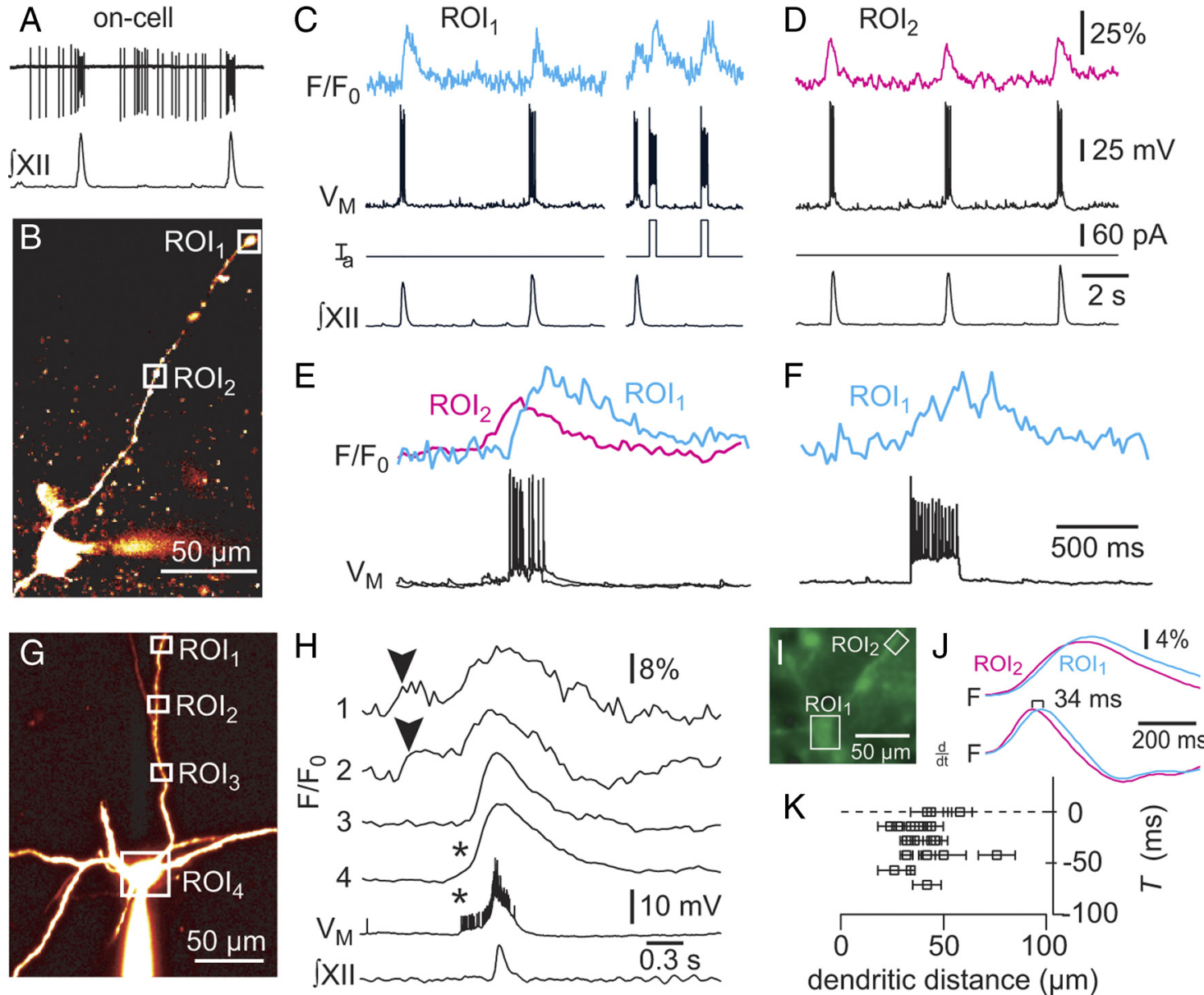

Figure 2. Dendritic $\mathrm{Ca}^{2+}$ measurements in preBötC neurons recorded with standard K-gluconate patch solution. $\boldsymbol{A}, 0 \mathrm{n}$-cell recording and XII output. $\boldsymbol{B}$, Image of the cell in $\boldsymbol{A}$ (Alexa 568). C, D, Dendritic $\mathrm{Ca}^{2+}$ dynamics in $\mathrm{ROI}_{1}(\boldsymbol{C})$ and $\mathrm{ROI}_{2}(\boldsymbol{D})$ with whole-cell and XII recordings. Step commands were applied while imaging ROI in $\boldsymbol{C}$. Vertical calibration bars apply to $\boldsymbol{C}-\boldsymbol{F}$. $\boldsymbol{E}$, The first cycles from $\boldsymbol{C}$ and $\boldsymbol{D}$ replotted and superimposed at higher sweep speed. $\boldsymbol{F}$, Second evoked response from $\boldsymbol{C}$ replotted at higher sweep speed. $\boldsymbol{G}$, Image of a different preBötC neuron (Alexa 568). $\boldsymbol{H}$, Cycle-triggered average of somatodendritic activity. Imaging sweeps for ROIs $1-4$ with membrane potential and XII output. Asterisks indicate somatic action potentials that evoked $\mathrm{Ca}^{2+}$ transients. Arrowheads (top traces) indicate negative dendritic $\mathrm{Ca}^{2+}$ latency. $\boldsymbol{I}-\boldsymbol{K}, \mathrm{Ca}^{2+}$ imaging from soma and dendrites. $\boldsymbol{I}$, Epifluorescence image. $\boldsymbol{J}$, Imaging sweeps showing raw and differentiated traces. $\boldsymbol{K}$, Dendritic $\mathrm{Ca}^{2+}$ latency versus recording distance for 20 dendritic recording sites. Horizontal error bars indicate the length of the dendritic ROI.

The patch solution contained $2 \mathrm{~mm}$ QX-314 to prevent voltage-gated $\mathrm{Ca}^{2+}$ channels evoked during action potentials from obscuring synaptic-dendritic $\mathrm{Ca}^{2+}$ activity. QX-314 blocks spiking intracellularly, but has no effect on network properties such as recurrent excitation, which can be detected as preinspiratory ramp-like voltage trajectory and temporal summation of EPSPs (Fig. 1B). Nevertheless, $\mathrm{Ca}^{2+}$ transients in the soma and proximal dendrites $(20-50 \mu \mathrm{m})$ coincided with the somatic inspiratory drive potential (Fig. $1 B, C$ ).

We hypothesized that postsynaptic $\mathrm{Ca}^{2+}$ accumulation before somatic burst generation might be detectable in more distal dendrites. To quantify the temporal relationship of dendritic $\mathrm{Ca}^{2+}$ and somatic inspiratory bursts, we subtracted the onset time of the inspiratory drive potential $\left(T_{\text {SOMA }}\right)$ from the fluorescence onset time in the dendrite $\left(T_{\mathrm{DEN}}\right)$ to obtain the dendritic $\mathrm{Ca}^{2+}$ latency $(\Delta T)$, which we dub drive latency. Figure $1, D$ and $E$, shows dendritic $\Delta F / F_{0}$ measured $90 \mu \mathrm{m}$ from the soma. $\Delta T$ was $<0$ in $75 \%$ of cycles measured; thus, dendritic $\mathrm{Ca}^{2+}$ generally increased before the inspiratory drive potential. The average dendritic $\mathrm{Ca}^{2+}$ latency measured $-12 \pm 8 \mathrm{~ms}$, with a range of -6 to $-34 \mathrm{~ms}(n=7$ cycles at $90 \mu \mathrm{m})$, and $-28 \pm 15 \mathrm{~ms}$ at an average distance of $76 \pm 15 \mu \mathrm{m}$ in five dendritic branches $(n=24$ cycles in total).
These latency times are reliable, even though they are smaller than the sampling interval ( $49 \mathrm{~ms} /$ frame), because $T_{\mathrm{DEN}}$ was determined based on the first dendritic measurement to exceed baseline fluorescence by two or more standard deviations; we did not interpolate between points to estimate $T_{\mathrm{DEN}}$. This method of computing $\Delta T$ may underestimate the dendritic $\mathrm{Ca}^{2+}$ latency because if the sampling rate was higher, a significant increase in dendritic $\mathrm{Ca}^{2+}$ fluorescence would be detectable earlier. The dendritic sampling rate has no effect on $T_{\mathrm{SOMA}}$, which is determined by electrophysiology $(10 \mathrm{kHz})$.

$\Delta T$ exceeded zero in two cycles (5 and $28 \mathrm{~ms}$, respectively) (Fig. $1 D$ ), which may reflect dendritic $\mathrm{Ca}^{2+}$ channel activity evoked electrotonically by somatic depolarization. To evaluate this possibility, we applied step-current commands $500 \mathrm{~ms}$ after the inspiratory burst, when network synaptic activity is minimal and few spontaneous synaptic potentials can be detected. The step command caused a significant dendritic fluorescence response within $38 \mathrm{~ms}$ (Fig. $1 F$ ), which was commensurate with inspiratory bursts in which $\Delta T$ was also positive and the dendritic $\Delta F / F_{0}$ occurred 5-28 ms after the inspiratory drive potential.

The peak dendritic fluorescence always occurred $\sim 200 \mathrm{~ms}$ (or more) after the onset of the drive potential, even during respiratory cycles in which initial dendritic $\mathrm{Ca}^{2+}$ preceded the somatic 
burst $(\Delta T<0)$ (Fig. $1 E)$. The dendritic fluorescence similarly peaked $\sim 240 \mathrm{~ms}$ after the step command (Fig. $1 F$ ). These data suggest that the maximum dendritic $\mathrm{Ca}^{2+}$ response is attributable to voltage-gated $\mathrm{Ca}^{2+}$ channels evoked electrotonically by somatic depolarization.

A caveat is that QX-314 also enhances the amplitude of the inspiratory drive potential (Pace et al., 2007a). AMPA receptors ordinarily evoke $\mathrm{Ca}^{2+}$ channels (Pace and Del Negro, 2008), but the QX-314-amplified drive potential may artificially increase $\mathrm{Ca}^{2+}$ channel recruitment and cause one to overestimate the synaptically triggered $\mathrm{Ca}^{2+}$ activity. Therefore, we subsequently omitted QX-314 from the patch solution and concentrated on measuring dendritic $\mathrm{Ca}^{2+}$ latency.

Figure 2, $A-F$, shows a preBötC neuron recorded with K-gluconate solution. Preinspiratory spiking was robust on-cell (Fig. 2A), so in whole-cell we applied negative bias to prevent intraburst spiking that would otherwise obscure our ability to measure synaptic $\mathrm{Ca}^{2+}$ activity. During inspiratory cycles, $\Delta T$ measured $87 \pm 13 \mathrm{~ms}$ at a site $220 \mu \mathrm{m}$ from the cell body $(n=22$ cycles; $\mathrm{ROI}_{1}$ ) (Fig. $2 \mathrm{~B}$ ). The latency at $\mathrm{ROI}_{1}$ was significantly longer in response to step-current injection through the somatic patch electrode (182 $\pm 35 \mathrm{~ms}, n=5$ pulse injections, $p<0.05$ ) (Fig. $2 C, F$ ). This disparity suggests that a proximal dendritic site triggered $\mathrm{Ca}^{2+}$ accumulation at $\mathrm{ROI}_{1}$ during inspiratory cycles. To test this, we recorded contiguous regions of the dendrite between $\mathrm{ROI}_{1}$ and the soma. At a site $81 \mu \mathrm{m}$ from the cell body, $\Delta T$ measured $-90 \pm 14 \mathrm{~ms}\left(n=23\right.$ cycles from $\left.\mathrm{ROI}_{2}\right)$ (Fig. 2D,E). Because $\mathrm{ROI}_{1}$ and $\mathrm{ROI}_{2}$ were separated by $139 \mu \mathrm{m}$, and $T_{\mathrm{DEN}}$ occurred $177 \mathrm{~ms}$ later at $\mathrm{ROI}_{1}$, the data suggest that $\mathrm{Ca}^{2+}$ activity originating at or near $\mathrm{ROI}_{2}$ influenced adjacent regions of the dendrite at a rate of $\sim 785 \mu \mathrm{m} / \mathrm{s}$.

We detected $\mathrm{Ca}^{2+}$ transients in the dendrite occurring both before and after the inspiratory burst in four experiments. Figure $2 G$ shows a cycle-triggered average in a representative preBötC neuron. Distal sites such as ROI ${ }_{1}$ and $\mathrm{ROI}_{2}$ (154 and $94 \mu \mathrm{m}$ from the soma, respectively) showed $\mathrm{Ca}^{2+}$ transients before the inspiratory phase, which propagated orthodromically at a rate in the range of $660-1000 \mu \mathrm{m} / \mathrm{s}$. $\mathrm{ROI}_{3}$ (57 $\mu \mathrm{m}$ from the soma) showed no evidence of $\mathrm{Ca}^{2+}$ activity before the inspiratory burst. Preinspiratory $\mathrm{Ca}^{2+}$ transients detected in the soma $\left(\mathrm{ROI}_{4}\right)$ were most likely attributable to preinspiratory spiking activity that we could not fully suppress via direct current bias (Fig. $2 \mathrm{H}$, asterisks). These data suggest that not all sites in the dendrite are excitable and that preinspiratory $\mathrm{Ca}^{2+}$ activity occurs in specialized hotspots. Peak fluorescence always occurred after the onset of the inspiratory phase, which is equally clear in Figures 1 and 2. $\mathrm{Ca}^{2+}$ transients that accompany the inspiratory burst occur synchronously throughout the dendrite, not in hotspots, and always peaked within $\sim 200 \mathrm{~ms}$ of $T_{\text {SOMA }}$ (Fig. $2 \mathrm{H}$ ).

We measured dendritic $\mathrm{Ca}^{2+}$ latency at 20 dendritic ROIs in eight preBötC neurons loaded by simultaneously imaging the dendrites and soma, which circumvents whole-cell dialysis that can affect the $\mathrm{Ca}^{2+}$ dynamics in the soma and dendrites. A representative experiment shows $\Delta T$ of $-34 \mathrm{~ms}$ for a dendritic site located $71 \mu \mathrm{m}$ from the soma (Fig. $2 I, J) . \Delta T$ measured $<0$ in 17 dendritic sites $24-74 \mu \mathrm{m}$ away from the soma and was $\sim 0$ in three cases (Fig. $2 K$ ).

We plotted the entire set of dendritic $\mathrm{Ca}^{2+}$ latency measurements for endogenous inspiratory bursts and responses to stepcurrent injection as a function of dendritic recording distance. These data include 36 recordings with K-gluconate patch solution (Fig. 3A, red), 17 recordings with QX-314 included (Fig. 3A, green), 20 different dendritic sites in eight neurons imaged at the
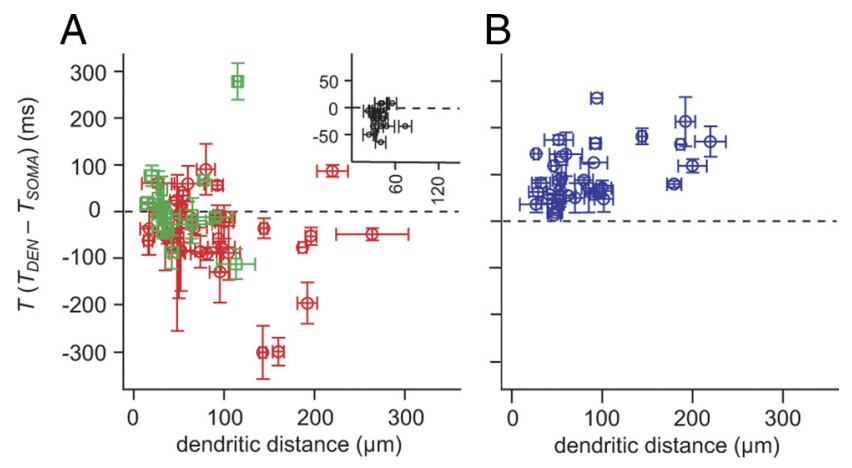

Figure 3. Plots of dendritic $\mathrm{Ca}^{2+}$ latency versus recording distance for the entire dataset. $\boldsymbol{A}$, Drive latency for endogenous cycles of respiratory activity (red, K-gluconate recordings; green, QX-314 experiments). Inset reproduces Figure 2 K. B, Dendritic $\mathrm{Ca}^{2+}$ latency in response to step commands (blue). Vertical error bars correspond to SE of $\Delta T$. Horizontal error bars indicate the length of the dendritic ROI.

soma and dendrite (Figs. $2 \mathrm{~K}, 3 \mathrm{~A}$, inset), and $\mathrm{Ca}^{2+}$ latency in response to step-current injection from 20 neurons (Fig. $3 B$ ). Points below the origin reflect dendritic $\mathrm{Ca}^{2+}$ activity that precedes inspiratory burst generation. Points above the origin reflect $\mathrm{Ca}^{2+}$ activity evoked electrotonically as a result of somatic or local dendritic depolarization. $\mathrm{Ca}^{2+}$ activity was detected before somatic burst generation in most experiments. However, at recording sites $<60 \mu \mathrm{m}$ from the soma, $\Delta T$ often exceeded zero, suggesting dendritic $\mathrm{Ca}^{2+}$ attributable to the electrotonic effects of somatic depolarization. The step-current injections universally evoked positive dendritic $\mathrm{Ca}^{2+}$ latency, as expected.

\section{Discussion}

The physiological properties of putatively rhythmogenic preBötC neurons - observed in vivo and in vitro over $\sim 30$ years-include a preinspiratory firing pattern and robust inspiratory bursts that experience intraburst depolarization block, which suggests the massive recruitment of inward current during inspiration (Richter, 1982; Rekling et al., 1996; Rekling and Feldman, 1998; Guyenet and Wang, 2001; Stornetta et al., 2003; Hayes and Del Negro, 2007; Rubin et al., 2009; Gray et al., 2010). We examined the $\mathrm{Ca}^{2+}$ signaling in dendrites to elucidate how the build-up of excitatory synaptic input evokes burst-generating conductances. In this study, the membrane properties of the presynaptic neurons remain unknown and could include a wide variety of discharge phenotypes, including neurons with bursting-pacemaker properties and intrinsic spiking activity, as well as neurons that participate in recurrent excitatory clusters. However, all the presynaptic neurons share a propensity to increase their activity during the early inspiratory phase.

We recorded preBötC neurons based on preinspiratory firing pattern and depolarization block (Figs. 1A, 2A), which strongly suggests glutamatergic phenotype (Gray et al., 2010). The other major subpopulations in the preBötC are inhibitory interneurons that display low-amplitude inspiratory bursts $(<5 \mathrm{mV})$ without preinspiratory firing (Kuwana et al., 2006; Winter et al., 2009).

Mironov $(2008,2009)$ documented dendritic waves of $\mathrm{Ca}^{2+}$. induced $\mathrm{Ca}^{2+}$-release (CICR) propagating at $72 \mu \mathrm{m} / \mathrm{s}$, triggered by metabotropic glutamate receptors in preBötC neurons. The ability to generate CICR waves may develop in culture or it may be limited to the proximal dendrite; we never detected similar $\mathrm{Ca}^{2+}$ waves in acute rhythmically active slices. We also recorded dendritic $\mathrm{Ca}^{2+}$ transients before somatic bursts, but not in every case. $\mathrm{Ca}^{2+}$ transients preceded somatic inspiratory bursts in 55 of 
74 experiments (74\%). In general, the more distal the recording site, the earlier we detected $\mathrm{Ca}^{2+}$ signals. What propagates from dendrite to soma: $\mathrm{Ca}^{2+}$ or voltage? CICR generally propagates at $\sim 70 \mu \mathrm{m} / \mathrm{s}$ in dendrites (Augustine et al., 2003). Alternatively, $\mathrm{Ca}^{2+}$ may evoke $I_{\mathrm{CAN}}$ at or near the synapse to depolarize the soma (and neighboring regions of dendrite) electrotonically. Cable theory shows that voltage changes propagate faster than CICR by orders of magnitude in neuronal dendrites. Since we did not detect $\mathrm{Ca}^{2+}$ waves per se, propagation reflects a quotient wherein the numerator is the distance between two recording sites and the denominator is the temporal disparity of burst onset at each site. According to this definition, we recorded propagation rates of $300-700 \mu \mathrm{m} / \mathrm{s}$, which is consistent with electrotonic propagation rather than CICR.

Dendritic $\mathrm{Ca}^{2+}$ transients originated after or in sync with somatic bursts or drive potentials in 19 of 74 experiments $(26 \%)$. Here too, the antidromic propagation rates were $300-700 \mu \mathrm{m} / \mathrm{s}$. Similar antidromic propagation rates were obtained in response to somatic step-current injections (Fig. $3 B$ ). The fact that their propagation rate is of similar magnitude (but opposite sign) to cycles with negative dendritic $\mathrm{Ca}^{2+}$ latency suggests that electrotonic factors govern the dendritic-somatic interactions in the preBötC in both orthodromic and antidromic responses. The data also suggest that some dendrites may receive very little or no drive at all, but still show $\mathrm{Ca}^{2+}$ activity by back propagating electrotonic effects.

We also detected dendritic sites with negative drive latency, whereas neighboring regions of the dendrite, both proximal and distal, showed either no $\mathrm{Ca}^{2+}$ activity $\left(\mathrm{ROI}_{3}\right)$ (Fig. $2 \mathrm{G}, \mathrm{H}$ ) or positive drive latency $\left(\mathrm{ROI}_{1}\right)$ (Fig. $2 B, C, E$ ). These data indicate that synaptic drive leading to postsynaptic $\mathrm{Ca}^{2+}$ elevation occurs in hotspots, which presumably indicates points at or near excitatory synaptic contacts. Sites with positive drive latency reflect $\mathrm{Ca}^{2+}$ channels activated electrotonically from the soma or neighboring regions of the dendrite, which again suggests electrotonic interactions among the dendrites and soma rather than CICR.

According to the group-pacemaker model, preBötC interneurons mutually interact to elevate postsynaptic $\mathrm{Ca}^{2+}$, activate the inward current $I_{\mathrm{CAN}}$, and periodically generate inspiratory bursts (Rekling et al., 1996; Rekling and Feldman, 1998; Mironov, 2008; Rubin et al., 2009). The relative timing of dendritic $\mathrm{Ca}^{2+}$ transients and somatic voltage changes is one measurable link between network-mediated synaptic drive and postsynaptic mechanisms that activate $I_{\text {CAN }}$ (Morgado-Valle et al., 2008). Although these data affirm the key prediction that postsynaptic $\mathrm{Ca}^{2+}$ transients be detectable before somatic bursts, they do not prove that respiratory rhythm is group-pacemaker driven. Nor can these data rule out alternative models that emphasize voltagedependent conductances such as persistent $\mathrm{Na}^{+}$current in rhythm generation (Koizumi and Smith, 2008). Regardless of whether emergent network properties constitute the core mechanism of respiratory rhythmogenesis, as the group-pacemaker model postulates, the synaptic activation of $I_{\mathrm{CAN}}$ contributes substantially to inspiratory burst generation (Johnson et al., 2007; Pace et al., 2007b) and is relevant to understanding the neural origins of respiratory rhythm.

We conclude that synaptic input typically triggers $\mathrm{Ca}^{2+}$ accumulation in dendrites before burst generation. Our measurements are inconsistent with regenerative $\mathrm{Ca}^{2+}$ propagation from dendrite to soma, which is a relatively slow process $(\sim 70 \mu \mathrm{m} / \mathrm{s})$ and unlikely to promote synaptic integration in the context of network rhythms. For example, consider an excitatory input 250 $\mu \mathrm{m}$ from the cell body. A CICR-dependent $\mathrm{Ca}^{2+}$ wave would require $3.5 \mathrm{~s}$ to influence the soma. In slices or en bloc preparations in vitro, the typical respiratory period is $\sim 5 \mathrm{~s}$. Synaptic inputs located $500 \mu \mathrm{m}$ (or farther) from the soma would be impractically sited to influence somatic integration during a typical respiratory cycle. Moreover, the slow propagation rate of CICR would prevent spatial and temporal summation of excitatory synaptic inputs that occur simultaneously at multiple positions in the dendritic arbor. Instead, synaptic inputs may elevate $\mathrm{Ca}^{2+}$, evoke $I_{\mathrm{CAN}}$, and depolarize the dendrite locally. These hotspots could thus act electrotonically to influence other regions of the dendrite and the soma in support of inspiratory burst generation. With propagation rates of $300-700 \mu \mathrm{m} / \mathrm{s}$, it would be reasonable for multiple dendritic sites to undergo temporal and spatial summation of inputs during a typical respiratory cycle in vitro, and even during breathing cycles in vivo in which cycle period is typically $<1 \mathrm{~s}$ in rodents.

\section{References}

Augustine GJ, Santamaria F, Tanaka K (2003) Local calcium signaling in neurons. Neuron 40:331-346.

Feldman JL, Del Negro CA (2006) Looking for inspiration: new perspectives on respiratory rhythm. Nat Rev Neurosci 7:232-242.

Gray PA, Hayes JA, Ling GY, Llona I, Tupal S, Picardo MC, Ross SE, Hirata T, Corbin JG, Eugenín J, Del Negro CA (2010) Developmental origin of preBötzinger complex respiratory neurons. J Neurosci 30:14883-14895.

Guyenet PG, Wang H (2001) Pre-Bötzinger neurons with preinspiratory discharges "in vivo" express NK1 receptors in the rat. J Neurophysiol $86: 438-446$.

Hayes JA, Del Negro CA (2007) Neurokinin receptor-expressing preBötzinger complex neurons in neonatal mice studied in vitro. J Neurophysiol 97:4215-4224.

Johnson SM, Wiegel LM, Majewski DJ (2007) Are pacemaker properties required for respiratory rhythm generation in adult turtle brain stems in vitro? Am J Physiol Regul Integr Comp Physiol 293:R901-R910.

Koizumi H, Smith JC (2008) Persistent Na+ and K+-dominated leak currents contribute to respiratory rhythm generation in the pre-Bötzinger complex in vitro. J Neurosci 28:1773-1785.

Kuwana S, Tsunekawa N, Yanagawa Y, Okada Y, Kuribayashi J, Obata K (2006) Electrophysiological and morphological characteristics of GABAergic respiratory neurons in the mouse pre-Botzinger complex. Eur J Neurosci 23:667-674.

Mironov S (2009) Respiratory circuits: function, mechanisms, topology, and pathology. Neuroscientist 15:194-208.

Mironov SL (2008) Metabotropic glutamate receptors activate dendritic calcium waves and TRPM channels which drive rhythmic respiratory patterns in mice. J Physiol 586:2277-2291.

Morgado-Valle C, Beltran-Parrazal L, DiFranco M, Vergara JL, Feldman JL (2008) Somatic Ca2 + transients do not contribute to inspiratory drive in preBötzinger complex neurons. J Physiol 586:4531-4540.

Pace RW, Del Negro CA (2008) AMPA and metabotropic glutamate receptors cooperatively generate inspiratory-like depolarization in mouse respiratory neurons in vitro. Eur J Neurosci 28:2434-2442.

Pace RW, Mackay DD, Feldman JL, Del Negro CA (2007a) Role of persistent sodium current in mouse preBotzinger complex neurons and respiratory rhythm generation. J Physiol 580:485-496.

Pace RW, Mackay DD, Feldman JL, Del Negro CA (2007b) Inspiratory bursts in the preBötzinger complex depend on a calcium-activated nonspecific cationic current linked to glutamate receptors. J Physiol 582:113-125.

Peña F, Parkis MA, Tryba AK, Ramirez JM (2004) Differential contribution of pacemaker properties to the generation of respiratory rhythms during normoxia and hypoxia. Neuron 43:105-117.

Ramirez JM, Tryba AK, Peña F (2004) Pacemaker neurons and neuronal networks: an integrative view. Curr Opin Neurobiol 14:665-674.

Rekling JC, Feldman JL (1998) PreBötzinger complex and pacemaker neurons: hypothesized site and kernel for respiratory rhythm generation. Annu Rev Physiol 60:385-405.

Rekling JC, Champagnat J, Denavit-Saubi é M (1996) Electroresponsive 
properties and membrane potential trajectories of three types of inspiratory neurons in the newborn mouse brain stem in vitro. J Neurophysiol 75:795-810.

Richter DW (1982) Generation and maintenance of the respiratory rhythm. J Exp Biol 100:93-107.

Ruangkittisakul A, Secchia L, Bornes TD, Palathinkal DM, Ballanyi K (2007) Dependence on extracellular $\mathrm{Ca} 2+/ \mathrm{K}+$ antagonism of inspiratory centre rhythms in slices and en bloc preparations of newborn rat brainstem. J Physiol 584:489-508.

Ruangkittisakul A, Panaitescu B, Ballanyi K (2010) K+ and Ca2+ dependence of inspiratory-related rhythm in novel "calibrated" mouse brainstem slices. Respir Physiol Neurobiol. Advance online publication. Retrieved September 15, 2010. doi:10.1016/j.resp.2010.09.004.
Rubin JE, Hayes JA, Mendenhall JL, Del Negro CA (2009) Calciumactivated nonspecific cation current and synaptic depression promote network-dependent burst oscillations. Proc Natl Acad Sci USA 106:2939-2944.

Stornetta RL, Sevigny CP, Guyenet PG (2003) Inspiratory augmenting bulbospinal neurons express both glutamatergic and enkephalinergic phenotypes. J Comp Neurol 455:113-124.

Thoby-Brisson M, Ramirez JM (2001) Identification of two types of inspiratory pacemaker neurons in the isolated respiratory neural network of mice. J Neurophysiol 86:104-112.

Winter SM, Fresemann J, Schnell C, Oku Y, Hirrlinger J, Hülsmann S (2009) Glycinergic interneurons are functionally integrated into the inspiratory network of mouse medullary slices. Pflugers Arch 458:459-469. 